\title{
TRANSFORMER INSULATING OILS
}

A USEFUL paper on transformer insulating oils was read by B. Calvert before the Sheffield Section of the Graduates and Students of the Institution of Electrical Engineers last year and is now printed in their Quarterly Journal.

Mr. Calvert points out that Faraday appears to have been the first to examine oily liquids for their insulating properties. The first commercial use made of his discoveries was in connexion with telegraph cables, for which rosin oil was employed. With alternating current transformers, the high temperatures used in practice made rosin oils unsuitable because of the formation of sludge and rapid evaporation of the oil. Thus modern transformers are usually immersed in mineral oil, which is freer from these disadvantages.

Quite recently a synthetic oil patented under the name of 'Pyranol' has been found excellent for insulating purposes and is extensively used in America. The crude oils from which insulating oils are derived can be divided into three types : first, those having a composition of more than 66 per cent of paraffins, that is, saturated open-chain fatty hydrocarbons, such as Pennsylvanian oils; secondly, those consisting of more than 66 per cent of naphthenes, such as Russian oils ; and thirdly, those having not more than two-thirds of either paraffins or naphthenes in their composition, such as Rumanian and Texas oils.

The insulating oils obtained from these crude oils are separated by fractional distillation and then purified with sulphuric acid; the tarry deposit resulting from this treatment is removed. The oil is then washed with caustic potash and water to remove any trace of acid. In order to remove the last impurities, such as fibres and dust, from the oil it is finally centrifuged and filtered before dispatch.

The cooling of a transformer depends principally upon the viscosity of the oil used, on which depends the convection currents; the transference of heat being more rapid with low viscosity. Unfortunately, as the viscosity decreases, flashpoint decreases and volatility increases. Thus a compromise has to be made since loss by evaporation is objectionable and fire risk should be as small as possible. Besides these properties, the oil must remain fluid at the lowest temperatures likely to be met with when the transformer is off load. The temperatures at which oils cease to be fluid vary considerably, some congealing at about the freezing point of water, others requiring a temperature lower than $-40^{\circ} \mathrm{C}$. Two further important attributes of a transformer oil are maximum resistance to the formation of sludge and acid when in service. In Great Britain the principal requirements have been defined and standardized by the British Standards Institution in specification No. 148/1933 entitled "Insulating Oils for Electrical Purposes other than Cables". It also describes the methods of test and the apparatus required.

The oil deteriorates under working conditions, and it is the manner and results of this deterioration that are of moment to the engineer using the transformers. From this point of view the most important characteristic is the variation of the electric strength. The presence of water and particles of solid matter in the oils have the effect of lowering the breakdown value. For example, a mixture of 4 c.c. of water with $3.74 \mathrm{mgm}$. of fibre in 10,000 c.c. of oil is sufficient to lower the electric strength of a clear dry oil from 100 kilovolts to 37 kilovolts, although both water and fibre are in a pure state. It should be noted that oil absorbs moisture from the atmosphere and therefore, if possible, the filling of transformers or other apparatus with oil should not be done on wet days. Pyranol is widely used in the United States, especially for indoor and traction work, because of its non-explosive and non-inflammable characteristies. Owing to the removal of the restrictions as to the use of fire-proof vaults, etc., the cost of the Pyranol-filled transformer is generally cheaper than the oil-filled installation. Owing to the high cost of pyranol, it is not thought that it will be widely used in Great Britain, at least for the present. Mr. Calvert thinks that there are cases where its use may be justified. In boiler houses air-cooled transformers have been installed owing to fire risks. In many cases pyranol transformers would be a better substitute, and on economic grounds alone would probably be justified.

\section{ELECTRIC STRENGTH OF SOME SOLID DIELECTRICS}

$\mathrm{I}^{\mathrm{N}}$ $\mathrm{N}$ connexion with the theory of the breakdown of solid dielectrics, the British Electrical and Allied Industries Research Association has made a helpful technical report* discussing important theories which have recently been advanced, and describing methods by which the 'intrinsic' electric strength of solid dielectrics may be defined and evaluated. It is shown that the magnitudes of, and the effect of temperature and thickness upon, the electric strength of certain crystals agrees with Fröhlich's theory of electronic breakdown. The effect of disordered structure and microstructure also agrees with it in

* Tech. Report, Ref. L/T 114: The Flectric Strength of Solid Dielectrics in relation to the Theory of Electronic Breakdown. Pp. $18+9$ plates. (London: British Electrical and Allied Industries Research Association.) 38. similar instances. On the other hand, departures from theory occur with complex organic dielectrics, and also with crystals when certain limits, for example, of the temperature of the material, are exceeded. Some observations are also made on the effect of temperature and electric stress upon the conductivity of the material.

The object of the present report is to amplify a previous note by A. E. W. Austen and W. Hackett (Nature, April 15, 1939; p. 637). Experimental values of the electric strength of certain solid dielectrics are compared with the values predicted theoretically from the known structure of the materials. The electrical breakdown is often influenced by the thermal and special electrical conditions 
under which the test is made; for example, the breakdown due to discharges in a surrounding medium or due to thermal instability. For the present purpose, the electric strength measured must be independent of such conditions. It appears that a type of breakdown which is a property only of the physical nature of the dielectric and its temperature exists, and may be defined. It has been called intrinsic breakdown, and the corresponding fiold strength is termed the intrinsic electric strength.

Intrinsic breakdown should possess the following properties : $(a)$ it should be independent of thickness over a fairly wide range ; $(b)$ it should be independent of the nature or duration of the electric stress, provided no appreciable change of temperature occurs as a result of the application of the electric stress, and provided the duration is sufficiently long ; $(c)$ the actual discharge should oceur wholly within the dielectric and in the region where the field is most intense. In the experiments it was found convenient to satisfy certain further conditions.

Attempts have been made to identify intrinsic breakdown with various mechanisms. Fröhlich has examined mathematically the equilibrium of an electron in a solid. His theory shows that beyond a certain eritical field-strength an electron may gain energy from the field, and will eventually ionize the lattice. The following deductions can be made from Fröhlich's theory : $(a)$ breakdown should increase with temperature for materials having a low Debye temperature, and be practically independent of temperature for substances with a high Debye temperature; $(b)$ the electric strength should be independent of thickness until the thickness approaches the order of the electronic mean free path, when the electric strength should increase with decrease of thickness.

Many interesting experimental results on mica and on glass, both plain and coloured, are given. The report eoneludes that it has been shown that the electric strength of certain solid dielectrics may be defined in such a way that it is independent of the immersion medium, if any, of the shape of the specimen, of the nature of the electrodes, of the nature of the electric stress provided the maximum value persists for a period of a few micro-seconds, and of the thickness, within certain limits. Excellent diagrams are given showing comparisons between experimental and calculated values, the agreement of the variation of conductance with temperature, and the electric strength of clear ruby muscovitemica.

Based on this report, a paper by A. E. W. Austen and $\$$. Whitehead on the same subject has been communicated to the Royal Society, and is published in the Proceedings (A) of August 28, 1940. It de. seribes some recent measurements of the intrinsic electric strength of certain solid dielectrics. This property of homogeneous solid dielectrics is defined, and methods of measurement suitable for a wide range of materials and conditions are described. A comparison of experimental results with Fröhlich's theory yields the following conclusions: (a) the electric strengths of mica, quartz, potassium bromide. and other alkali halides agree with the theory; $(b)$ the effect of temperature on the electric strengths of mica and potassium bromide agree qualitatively with the theory, while for mica the agreement is also satisfactory quantitatively; $(c)$ the electric strength of mica increases when the thickness is reduced to the order of a few mean free paths, and the agreement is quantitative to the aceuracy with which calculation is possible; (d) fused quartz has a relatively higher electric strength than crystalline quartz, corresponding to the prediction of the theory that disorder increases the electrie strength.

\section{YELLOW FEVER AND ITS CONTROL*}

$\mathrm{I}^{\mathrm{N}}$ research on yellow fever to-day, the tiny chick embryo, both as minced tissue culture and as the whole embryo in the shell, is one of the most important of the experimental animals used; but man himself was the only useful experimental animal in the study of this disease until as recently as 1927. Research was thus impeded until that time by the reluctance to expose human volunteers to so dangerous an experimental infection.

The extensive history of attempts to learn how the disease was transmitted reveals, nevertheless, that numerous medical men, without thought to their own comfort or safety, performed the most umpleasant experiments upon themselves. A medical student of the University of Pennsylvania during 1802-1803 placed black vomit and blood serum from yellow fever patients in wounds made in his arms and legs and injected black vomit into animals. Other early experimenters exposed their skins to the soiled clothing, bedding, sweat, black vomit, tissues or blood of vellow fever patients.

In none of these experiments did the yellow fever 'take', and the evidence therefore seemed strong that the disease was not contagious. However, there was so much interest in the disease that a total of

* From a paper by Dr. Wilbur A. Sawyer read on September 17 at the Bicentennial Conference of the Uiniversity of Pennsylvania.
556 articles on it are referred to by a writer of 1827 , reviewing the literature since 1797 .

Although generally accepted proof that a mosquito was the carrier of the disease was not established until the work of Dr. Walter Reed and his associates of the U.S. Army Yellow Fever Commission in 1900 and 1901, Dr. Carlos J. Finlay, of Havana, declared as early as 1881 that he was convinced a mosquito was responsible; and he pointed to the right species, namely, Aerles aegypti. Finlay used 102 human volunteers, who permitted themselves to be bitten by mosquitoes which had sucked the blood of yellow fever patients. That the few illnesses observed among his volunteers could not be accepted as yellow fever is due, Dr. Sawyer suggests, to the fact that Finlay did not let the virus incubate long enough in the mosquitoes before they bit the volunteers.

After Reed's brilliant work showed the way to successful control of yellow fever by eliminating the mosquito, no important new discoveries about the disease were made for another quarter century. This was partly because of the deaths of several human volunteers and the reluctance to use more.

Interest in the possibility of immunization was aroused when, in 1927, Drs. A. Stokes, J. H. Bauer and N. P. Hudson, while making investigations in West Africa, discovered that Asiatic monkeys. 